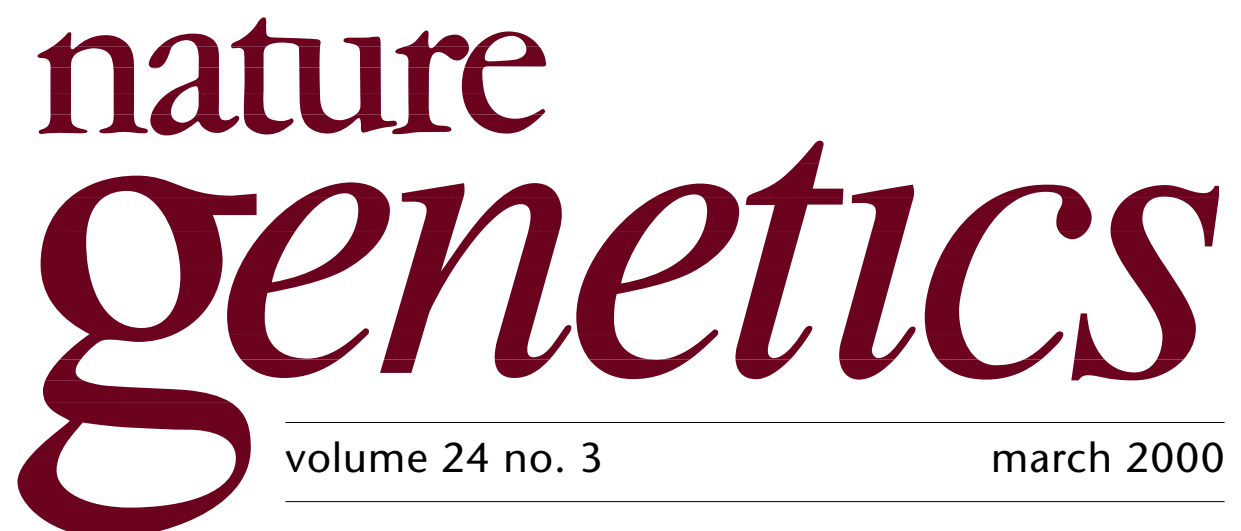

\title{
Trials and tribulations
}

This issue includes a report (page 257) of a phase I clinical trial, a type of paper rarely seen in Nature Genetics. The protocol involves gene therapy-a subject of heated debate since the tragic death of Jesse Gelsinger, the young man who died after being administered a large dose of adenoviral vector in September 1999. Katherine High and colleagues, the authors of the present study, injected adenoassociated vector carrying the gene encoding factor IX into the skeletal muscles of three patients with severe haemophilia B. As the first participants in a trial of doseescalation design, the three patients received the lowest dose of the vector, $2 \times 10^{11}$ vector genomes per kilogram body weight. On the basis of preclinical data from animal models, this dose was not expected to increase the levels of circulating factor IX. In two of the patients, however, there was evidence of a modest clinical response as measured by a $50 \%$ to $80 \%$ reduction of episodes of factor infusion and by changes (albeit small) in levels of circulating factor IX. A third patient, who has the least severe phenotype, showed no measurable differences after injection.

Why is Nature Genetics publishing this study? Although it is preliminary, it nevertheless reports modest evidence of efficacy at low doses of virus, and it may prove to be the first report of clinically efficacious application of gene therapy to haemophilia. Subsequent studies confirming absence of toxicity and increased efficacy of higher doses of the vector will determine whether the protocol lives up to this potential. The study is exemplary in other aspects: similar protocols in two different and highly suitable animal models (transgenic mice and haemophilic dogs) had shown absence of vector-related toxicity and efficacy of the treatment, justifying the step into the clinic. Arthur Beaudet, a gene-therapy researcher at Baylor College of Medicine in Houston, comments: "it is gratifying to see the very attractive study design (sensitive tests for factor activity, very low risk and quantitating self-administration of factor) to provide a favourable risk-benefit ratio. Internal review boards, the Recombinant DNA Advisory Committee, the Food and Drug Administration and, most of all, investigators - as they are inevitably the most informed-should ask if any research protocol meets the litmus test of whether you would enroll yourself or your loved one in this protocol given the same circumstance. This study meets that test."

As with the protocol in which Jesse Gelsinger was enrolled, it is a phase I trial, designed to determine safety; most phase I trials do not yield data on efficacy (see box). In this particular case, however, the existence of defined short-term clinical endpoints allowed the investigators to collect preliminary data on efficacy. By definition, therapy administered during phase I trials cannot be considered to be a 
proven treatment, and is therefore unlikely to be of real benefit to the patient. A disturbing issue that has surfaced in the aftermath of Gelsinger's death concerns informed consent and the differing inspirations of the researcher and the patient: one seeks to ascertain the safety of experimental treatment, whereas the other hopes for a cure.

Federal regulations require that patients are informed of the risks and benefits of an experiment, but some experts argue that consent forms frequently exaggerate the benefits. According to bioethicist LeRoy Walters, former chairman of the Recombinant DNA Advisory Committee, "consent forms are often deficient and they overpromise. They make phase I trials sound like the cure for your cancer" (The New York Times, 27 January 2000). Some clinical researchers disagree, but assessing the extent to which consent really is informed requires hard data, rather than anecdotes. In the United States, patient enroll-

\section{Clinical trials}

Phase I: Represent first studies in humans and collect data on dosage, timing and safety-but not efficacy-of an investigational treatment in a small number of subjects. The trials typically involve the gradual escalation of dosage to determine the maximum tolerable level. Data are also collected on how a drug is absorbed, metabolized and distributed through the body.

Phase II: Continued evaluation of the safety of the treatment as well as its efficacy according to specific parameters in a larger group of patients.

Phase III: Gather large-scale efficacy data from large numbers of patients to determine whether the treatment under study is better than the current standard of care and to compare side effects. Phase III trials are usually randomized and involve a greater diversity of patients (according to such variables as gender, age and race). ment is a major limiting factor in clinical trials. Encouraging participation by playing down the risks and exaggerating the potential benefits, however, must not be the solution.

Concern regarding the risk-to-benefit ratio is not the only reason that dissuades patients from participating in trials. According to a survey conducted by the American Society for Clinical Oncology, major deterrents for physicians to enroll patients in clinical trials are additional costs and uncertainty about reimbursement, extensive paperwork and narrow eligibility criteria (only a fraction of the patients who seem eligible turn out to be upon closer inspection, causing disappointment after hopes have been raised). For patients, major barriers are inconvenience (many receive treatment at locations distant to their homes) and uncertainty about insurance coverage. An analysis by the National Cancer Institute showed that the cost of treating a cancer patient enrolled in a clinical trial is not dramatically higher than the cost of standard treatment. As society depends on clinical trials to optimize and update standard medical praxis, and clinical trials depend upon the participation of patients, it is imperative to mandate insurance coverage of the costs-especially as this is unlikely to change health care economy in a major way.

Clinical trials—including gene therapy trials—-must adhere to strict standards and protocols. At the same time, approval mechanisms must be timely so that the science upon which trials are based is not outdated by the time the first patients receive treatment. Researchers in academia and industry are concerned that Gelsinger's death could further compromise enrollment in clinical trials, and that additional layers of regulation - which would inevitably be associated with more paperwork and prolonged approval times - could be introduced. Responsible physicians and scientists will not object to full disclosure of the risks and benefits of clinical trials, random control visits by Federal Drug Administration or National Institutes of Health inspectors, or stricter enforcement of existing regulations. Painting a more negative picture of clinical trials in general and additional layers of regulation, however, will jeopardize the efficient translation of basic research into clinical practice - at a time when many therapies are finally based on an understanding of the underlying defects rather than trial and error.

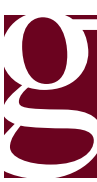

